\title{
Leveraging diversity, equity and inclusion for promoting wellness in the radiology workplace
}

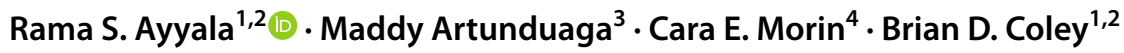

Received: 22 July 2021 / Revised: 11 December 2021 / Accepted: 18 January 2022 / Published online: 28 January 2022

(c) The Author(s), under exclusive licence to Springer-Verlag GmbH Germany, part of Springer Nature 2022

\begin{abstract}
Over the last decade, health care professionals in the field of radiology have experienced increasing rates of burnout. A study in 2017 showed high prevalence of burnout in pediatric radiology, and other studies have identified several drivers for burnout. An important factor in promoting wellness and mitigating burnout is leveraging diversity, equity and inclusion in the workplace. This manuscript highlights the importance of diversity in high-functioning teams as well as the critical role of equity and inclusion in the workplace to help create an organization where people belong and can effectively succeed.
\end{abstract}

Keywords Diversity $\cdot$ Equity $\cdot$ Inclusion $\cdot$ Pediatric radiology $\cdot$ Wellness $\cdot$ Workplace

\section{Introduction}

Occupational burnout is defined as a psychological syndrome emerging as a prolonged response to chronic stressors on the job. Maslach et al. [1,2] defined three dimensions of burnout: emotional exhaustion (feeling overextended and depleted), depersonalization (cynicism and detached/callous attitude toward one's job) and lack of perceived accomplishment (feeling of not doing a job to one's fullest capabilities). Burnout in medicine has become a growing issue over the last decade. In a 2021 Medscape survey, almost $50 \%$ of physicians reported burnout [3]. Consistent with this, those working in radiology reported a high prevalence of burnout, with variability among subspecialties [4-6]. Although the 2021 Medscape survey (an annual survey of all medical subspecialists on wellness and burnout) showed that overall

Rama S. Ayyala

Rama.ayyala@cchmc.org

1 Department of Radiology,

Cincinnati Children's Hospital Medical Center,

3333 Burnett Ave., Cincinnati, OH 45229, USA

2 Department of Radiology, University of Cincinnati College of Medicine, Cincinnati, OH, USA

3 Department of Radiology, Pediatric Radiology Division, UT Southwestern Medical Center/Children's Health Medical Center, Dallas, TX, USA

4 Department of Diagnostic Imaging, St. Jude Children's Research Hospital, Memphis, TN, USA burnout had started trending down for radiologists, there is concern that new stresses and anxieties related to the challenges imposed by the coronavirus disease 2019 (COVID19) pandemic might reverse this trend [3]. A pediatric radiology investigation in 2017 showed that $66 \%$ of attending physicians reported burnout [7], with potential drivers including call burden, challenges with work-life imbalance and concerns regarding financial reimbursements [8]. Additional stressors associated with burnout include increased clinical demands and administrative tasks, which ultimately impact job satisfaction and have significant mental health implications [9].

Before the COVID-19 pandemic, potential drivers of burnout in radiology were identified, and some thought that they were heightened in our specialty compared to other medical fields. Examples of drivers included isolation, lack of a sense of community, and perceived ineffective leadership $[10,11]$. Many of these factors have worsened during the COVID-19 pandemic. A study identified areas of potential pandemic-related stress and anxiety among internal medicine physicians. Respondents endorsed concerns related to the overall uncertainty of the COVID-19 situation, selfprotection from the virus while effectively providing patient care, and adequate support for non-work-related stresses such as childcare [12]. A survey evaluating stresses and anxieties in pediatric radiologists during the early phases of the pandemic mirrored these findings. One important finding was gender-related differences in female pediatric radiologists experiencing higher overall stress and anxiety related 
to the challenges imposed by COVID-19 and increased guilt related to lower virus exposure compared to that for other radiology staff, in comparison to male colleagues [13].

Limited data show differences in rates of burnout in various physician demographics such as gender, age and race/ ethnicity. Female physicians are 1.6 times more likely to self-report burnout compared to male physicians [14]. Garcia et al. [14] found that physicians in underrepresented racial/ethnic groups (Hispanic/Latino/Latinx, non-Hispanic Black, and non-Hispanic Asian) reported burnout less frequently than did non-Hispanic White physicians; however, there are limitations to that study that question the validity of results, specifically the sample size and analysis of associated factors [15]. Another investigation found that among medical students, those from underrepresented groups reported a lower sense of personal accomplishment (one of the three dimensions of burnout defined by Maslach) [16]. Further studies are needed to evaluate these differences in medicine.

Physician wellness and burnout are not mutually exclusive opposites; therefore, mitigating burnout does not in itself lead to physician wellness. Wellness encompasses not only the absence of burnout, but also having an overall good quality of life, which involves - but is not exclusive to - being engaged at work, having a sense of meaning and purpose, and being able to balance work and non-work duties [17]. For promoting physician wellness, a multifaceted approach is needed that includes mitigation strategies to counter physician burnout. One important strategy is to leverage workplace diversity, equity and inclusion. Our manuscript highlights this topic and recommends potential areas to focus on to accelerate wellness.

\section{The link among diversity, equity, inclusion and wellness}

"Diversity is having a seat at the table, inclusion is having a voice, and belonging is having that voice be heard." This quote by Liz Fosslien and Mollie West Duffy [18] perfectly describes how simply promoting diversity is not enough: one needs to go further to foster inclusion and belonging. Without inclusion, diversity efforts are ineffective. There are many potential ways by which diversity, equity and inclusion in the workplace can improve physician wellness. The discrepancy in diversity in medicine, and specifically in radiology, is highlighted in other publications in this supplement. Aspects of diversity include traditional demographic factors including age, gender, race, ethnicity and sexual orientation. However, other less commonly considered categories include ableness, gender expression, generation and years in practice/previous practice experience. Focusing on improving workplace diversity can have many implications. Herein, we focus on how diversity plays a role in wellness in the workplace and can have both individual and institutional/ organizational benefits.

A 2018 Association of American Medical Colleges (AAMC) workforce survey showed that $56.8 \%$ of physicians in the United States are White and $65.5 \%$ are male [19]. In radiology, the gender imbalance is even greater at $73.2 \%$ male [20]. Given that White men are the largest cohort of physicians, many departments/institutions might predominantly be composed of this dominant group. Sometimes an underrepresented member might be one of the few or an "only," leading to a feeling of being a token member of the group or a part of a group for metric measures only. The creation of an "only" might not be intentional on the part of a department/practice but rather a byproduct of upstream issues and lack of an effective pipeline to change the environment. The feeling of being excluded as the least represented person in a group can heighten feelings of inherent biases and make one feel more scrutinized and held to higher and different standards than others. The "only" status can be double when one is not just the only woman but also the only person of a race/ethnicity in the group. This can lead to pressure to perform better and to be on guard, as well as more bias and discrimination, which can contribute to a sense of loneliness and being left out [21]. Contrary to being just a perception, one might actually have less access to resources, meetings and networking to facilitate career success. For example, the only female non-White radiologist in a practice might not have access to a network that her male colleagues have, and less access to mentorship or a sponsorship that would expose her to the broader medical community for obtaining the developmental opportunities needed to flourish and progress. Research has shown that men and women network differently, which can lead to fewer opportunities for women because of these inherent differences; for example, women might not attend after-work networking events so they can return home to care for family [22]. This can limit an individual's success, hinder advancement, delay promotion and eventually affect personal wellness. One of the three dimensions of burnout is perceived lack of accomplishment. This dimension is heightened for those who might not have access to the same opportunities (see the example above). It is important to create a psychologically safe environment for the "only" individuals to foster inclusivity, which can in turn develop into an effective pipeline to recruit a diverse workforce. This then would give a sense of accomplishment and improve overall job satisfaction for the individual as well as the collective group. In smaller departments, this can be challenging and disproportionately burdensome than in larger departments. Therefore, it is important to address and prioritize this not only at the departmental level, but also within institutions/organizations. Ultimately, individual wellness can impact workplace wellness. A non-inclusive 
work environment can lead to increased absenteeism, which can eventually result in a pipeline leakage by driving people to leave a position, or sometimes an entire career [23].

Another dimension of burnout is emotional exhaustion, which is a state of feeling drained from your professional responsibilities. Health care professionals in radiology have had a higher incidence of working in isolation, especially during the COVID-19 pandemic [24]. The lack of social connection and engagement caused by isolation heightens loneliness, which has been linked to detrimental physical and mental health effects [25]. On the other hand, social connectedness leads to increased self-esteem and confidence, which contributes to employees being more trusting, empathetic and cooperative, and therefore more effective [26]. Engagement at work is key for high-functioning teams to be successful. Employees with the highest levels of engagement demonstrate increased productivity and are less likely to leave their organization [27]. Organizations with top quartile racial and ethnic diversities are 35\% more likely to have financial performance above medians; in addition, organizations with more diverse workforces have higher rates of employee retention because individuals more often feel accepted and valued [27]. More diverse organizations can rely on a greater range of experiences and backgrounds to create sustainable initiatives [28]. This can help build a workplace that creates a more inclusive culture, which can in turn attract more diverse employees, thereby developing a potential pipeline.

\section{What needs to be done to improve diversity, equity, inclusion and wellness?}

Although the drivers of burnout in medicine and radiology are multifactorial, diversity plays a large role in workplace wellness, ultimately impacting individual wellness. Besides initiatives for increasing diversity in radiology, such as focusing on the radiology pipeline and mitigating potential leaks, along with increasing awareness and education on the topic, the following specific strategies need to be focused on for optimizing equity and inclusion in departments and institutions to promote wellness (Table 1):

1) developing and fostering high-functioning teams,

2) building an inclusive work environment and

3) emphasizing and fostering mentorship, sponsorship and allyship.

\section{Developing high-functioning teams}

High-functioning teams are a crucial component of an effective and successful workplace. Teams with diverse opinions, viewpoints, perspectives and backgrounds outperform homogeneous groups in problem-solving tasks [29]. This includes diversity on the basis of a wide range of factors such as age, gender, experience, knowledge, race, ethnicity

Table 1 Strategies for optimizing equity and inclusion in departments and institutions to promote wellness

\begin{tabular}{|c|c|c|}
\hline Actions & Attributes & Implementation examples \\
\hline \multirow[t]{3}{*}{ Develop and foster high-functioning teams } & $\begin{array}{l}\text { Diversity of } \\
\text { representa- } \\
\text { tion }\end{array}$ & Ensuring leadership and committees include diverse backgrounds and perspectives \\
\hline & Transparency & Providing clarity regarding policies, procedures, schedules and decision-making \\
\hline & Collegiality & $\begin{array}{l}\text { Conducting formal surveys and meetings and informal "check-ins" to identify pain } \\
\text { points and co-create solutions }\end{array}$ \\
\hline \multirow[t]{3}{*}{ Build an inclusive environment } & Education & $\begin{array}{l}\text { Understanding the breadth of diversity through formal and self-education about } \\
\text { visible and invisible aspects of diversity }\end{array}$ \\
\hline & Awareness & $\begin{array}{l}\text { Accommodating and advocating for situational needs (e.g., pumping rooms, reli- } \\
\text { gious accommodations) }\end{array}$ \\
\hline & Belonging & $\begin{array}{l}\text { Intentionally being inclusive of all opinions and voices, with specific attention to } \\
\text { conducting important meetings during work hours and minimizing after-work } \\
\text { obligations that exclude some }\end{array}$ \\
\hline \multirow[t]{3}{*}{$\begin{array}{l}\text { Create a culture of personal support and } \\
\text { professional development }\end{array}$} & Mentorship & $\begin{array}{l}\text { Providing guidance on job transitions, creating career development plans and } \\
\text { professional challenges }\end{array}$ \\
\hline & Allyship & $\begin{array}{l}\text { Advocating for underrepresented groups (e.g., a female colleague to be considered } \\
\text { for a leadership position in the department) } \\
\text { Being vocal against discriminatory or disparaging comments against colleagues }\end{array}$ \\
\hline & Sponsorship & $\begin{array}{l}\text { Using one's influence and network to invest in someone else's career success (e.g., } \\
\text { suggesting a colleague for an invited talk or nominating a colleague for a national } \\
\text { committee leadership role or offering a colleague as a replacement for an opportu- } \\
\text { nity offered to you - using your "no" for another person's "yes") }\end{array}$ \\
\hline
\end{tabular}


and skill sets. Another area that is important is cognitive diversity, which is defined as differences in perspective or information-processing styles, which is independent of other demographic factors of diversity [30].

There are two main advantages to fostering high-functioning teams in medicine: (1) improvement in quality of patient care and (2) positive impact on professional fulfillment of the workforce. Creating a collegial and collaborative work environment helps with workplace wellness because team dynamics can affect individual burnout. Burnout can be transmitted from one team member to another, and higher levels of aggregate team burnout have been linked to adverse patient outcomes [31]. A prerequisite for creating high-functioning teams is fostering a safe and inclusive environment so that views of all members can be heard and contribute to the diversity of thought, which results in better department decisions and performance.

\section{Building an inclusive work environment}

A key component of wellness in the workplace is creating a culture of inclusion and empathy. Given that many groups in medicine and radiology are relatively homogeneous, workplaces need to ensure that each individual is accepted, recognized and provided a safe space to be included. This can pose challenges, given that diversity can exist on many levels, some of which might not be visible, such as generational, cultural or religious. Therefore, an inclusive workplace needs to account for different viewpoints and expectations. This requires education and recognition of intersectionality (how different aspects of one's life can overlap to contribute to a person's lived experience) to create effective institutional and practice initiatives for a transparent and inclusive culture.

Situational and inclusive awareness is important to understand the basis for developing a better workplace culture. Situational awareness refers to being aware of the workplace dynamics to determine how differences among coworkers might play a role in making one feel less included [32]. Muslim coworkers who need to pray multiple times a day might feel uncomfortable bringing this up in a department, especially if this takes them away from clinical responsibilities. A new mother who recently returned to work and needs to pump breastmilk throughout the day might not feel comfortable telling her predominantly male colleagues that she needs to excuse herself from clinical duties. When few women or only one woman work in a predominantly male department where subtle comments that are thought to be harmless actually make the women uncomfortable, they might be uneasy to raise the issue. These examples highlight the importance of paying attention, being aware and embracing different viewpoints to create a culture of respect for all. Inclusive awareness is needed to create a safe and open work environment for all colleagues and patients. This includes supporting all gender identities by creating a trans-inclusive work environment. Potential methods of doing this include avoiding heteronormative assumptions (e.g., using preferred pronouns as descriptors of an individual), creating availability of gender-neutral bathrooms, as well as continuing education and training on the various aspects of gender diversity [33]. In the same way, having policies and practices that support an identity-safe environment are important, such as antidiscrimination and family leave policies [34]. An inclusive work environment plays a significant role in fostering best performances and helps individuals grow, especially by creating psychological and emotional safety at the workplace for those seeking mentorship, allyship and sponsorship.

\section{Fostering mentorship, allyship and sponsorship}

Mentorship, allyship and sponsorship have been shown to help all individuals continue to thrive and advance their careers [35]. However, individuals from underrepresented groups have less access to these resources than those in the majority. Research shows that White male leaders are not comfortable reaching out to young women or other underrepresented individuals to be a potential mentor or sponsor [36]. To create a more inclusive workplace with diversity at all levels, it is crucial to foster mentorship, allyship and sponsorship for all.

It is important to note the distinction among mentors, allies and sponsors. Mentors are experienced and trusted advisers who can offer advice and insights to grow and advance one's career. Mentors play critical roles at various stages of life and can empower one to explore options that were previously not considered, give access to previously untapped opportunities and help a person navigate difficult situations [37]. Allies are proactive supporters, collaborators and accomplices, commonly in privileged positions and not from under-represented groups, who help one achieve goals. Sponsors are champions who are invested in another's success and are willing to use their assets and connections to further grow and advance the other person's career [35]. One or several individuals in a department/institution can serve in all three roles, or a combination, for different people or situations. Mentors, sponsors and allies can play a crucial role in increasing inclusion/belonging in a department or institution to help with an individual's growth and wellness. The key is for all members of a department to have equal access to reliable people in all three roles, especially early in their careers. It is vital for departments and institutions to encourage and facilitate these relationships, which might not occur naturally.

For a successful mentoring program, it is important to foster both mentors and mentees. Potentially effective 
mentors need to be identified and trained. Mentees need help identifying and connecting with mentors (among different arenas, when applicable), and also guidance in nurturing and maintaining their relationship with the mentor [37]. Through these programs, larger networks of contacts can be generated, which can lead to greater opportunities for sponsorship. However, this can be particularly challenging for women and underrepresented groups because those in leadership positions are likely to be from a majority group, most commonly White men. It is important for those in this group to acknowledge their inherent privilege and opportunities, and therefore educate themselves and accept feedback to become better allies for those in underrepresented groups [38]. Being aware of this discrepancy in the availability of mentorship, allyship and sponsorship in underrepresented groups is important to foster the creation of a potential pool of resources, which can help with continuously fostering these relationships for future generations. Creating a diverse mentor/sponsor pool might also involve reaching outside the institution/department to connect an individual to the best matches [39]. Allyship is especially important for underrepresented groups, including women, because dominant groups can be vocal advocates, mentors and sponsors [32]. These connections and support can also help one navigate difficult situations, insecurity in the workplace, or feelings of exclusion, and can ultimately lead to an improved work culture and faculty retention. The benefits should not be just individual but global in a department/institution [35].

\section{Conclusion}

Many factors and drivers play a role in mitigating burnout and promoting wellness in the workplace. However, one of the most critical areas of focus is emphasizing the importance of diversity, equity and inclusion in radiology departments and institutions. In conjunction with education on the importance of diversity and equity, as well as initiatives to improve the pipeline for an overall more diverse radiology workforce, inclusivity plays a key role in creating an optimal workplace culture and fostering both institutional and individual wellness. It is vital for radiology departments to invest in creating these work environments that foster highfunctioning teams and promote the development of strong networks of mentors, sponsors and allies to optimize belonging, and eventually productivity, fulfillment and happiness.

\section{Declarations}

Conflicts of interest None

\section{References}

1. Maslach C, Jackson S (1981) The measurement of experienced burnout. J Occup Behav 2:99-113

2. Maslach C, Jackson S, Leiter M (1996) The Maslach burnout inventory manual. Consulting Psychologists Press, Palo Alto

3. Kane L (2021) "Death by 1,000 cuts": Medscape national physician burnout \& suicide report 2021. Medscape, New York

4. Chew FS, Mulcahy MJ, Porrino JA et al (2017) Prevalence of burnout among musculoskeletal radiologists. Skelet Radiol 46:497-506

5. Parikh J, Sun J, Mainiero M (2020) Prevalence of burnout in breast imaging radiologists. J Breast Imaging 2:112-118

6. Chen JY, Lexa FJ (2017) Baseline survey of the neuroradiology work environment in the United States with reported trends in clinical work, nonclinical work, perceptions of trainees, and burnout metrics. AJNR Am J Neuroradiol 38:1284-1291

7. Ayyala RS, Ahmed FS, Ruzal-Shapiro C, Taylor GA (2018) Prevalence of burnout among pediatric radiologists. J Am Coll Radiol 16:518-522

8. Ayyala RS, Ahmed F, Ruzal-Shapiro CB, Taylor G (2019) Stressors contributing to burnout amongst pediatric radiologists: results from a survey of the Society for Pediatric Radiology. Pediatr Radiol 49:714-722

9. Ayyala RS, Baird GL, Sze R et al (2020) The growing issue of burnout in radiology - a survey-based evaluation of driving factors and potential impacts in pediatric radiologists. Pediatr Radiol 50:1071-1077

10. Harolds JA, Parikh JR, Bluth EI et al (2016) Burnout of radiologists: frequency, risk factors, and remedies: a report of the ACR Commission on Human Resources. J Am Coll Radiol 3:411-416

11. Kruskal JB, Shanafelt T, Eby P et al (2019) A road map to foster wellness and engagement in our workplace - a report of the 2018 summer intersociety meeting. J Am Coll Radiol 16:869-877

12. Shanafelt T, Ripp J, Trockel M (2020) Understanding and addressing sources of anxiety among health care professionals during the COVID-19 pandemic. JAMA 323:2133-2134

13. Ayyala RS, Baird G, Bloom D et al (2021) Evaluation of stress and anxiety due to the coronavirus disease 2019 (COVID-19) pandemic in pediatric radiology. Pediatr Radiol 51:1589-1596

14. Garcia LC, Shanafelt TD, West CP et al (2020) Burnout, depression, career satisfaction, and work-life integration by physician race/ethnicity. JAMA Netw Open 3:e2012762

15. Cantor JC, Mouzon DM (2020) Are Hispanic, Black, and Asian physicians truly less burned out than White physicians? Individual and institutional considerations. JAMA Netw Open 3:e2013099

16. Dyrbye LN, Thomas MR, Huschka MM et al (2006) A multicenter study of burnout, depression, and quality of life in minority and nonminority U.S. medical students. Mayo Clin Proc $81: 1435-1442$

17. Eckleberry-Hunt J, Van Dyke A, Lick D, Tucciarone J (2009) Changing the conversation from burnout to wellness: physician well-being in residency training programs. J Grad Med Educ 1:225-230

18. Fosslien L, West Duffy M (2019) No hard feelings: emotions at work (and how they help us succeed). Penguin Publishing, New York

19. Association of American Medical Colleges (AAMC) (2019) Diversity in medicine: facts and figures 2019. https://www.aamc. org/data-reports/workforce/interactive-data/figure-20-percentagephysicians-sex-and-race/ethnicity-2018. Accessed 11 Jan 2022

20. Murphy B (2019) These medical specialties have the biggest gender imbalances. American Medical Association. https://www.amaassn.org/residents-students/specialty-profiles/these-medical-speci alties-have-biggest-gender-imbalances. Accessed 11 Jan 2022 
21. No authors listed (2021) Women in the workplace 2021. McKinsey \& Company. https://www.mckinsey.com/featured-insights/ diversity-and-inclusion/women-in-the-workplace. Accessed 11 Jan 2022

22. Castrillon C (2019) Why women need to network differently than men to get ahead. Forbes. https://www.forbes.com/sites/carolineca strillon/2019/03/10/why-women-need-to-network-differentlythan-men-to-get-ahead/?sh=1f557a2bb0a1. Accessed 11 Jan 2022

23. Pierce J (2019) Minimizing employee turnover in a changing workforce. Forbes. https://www.forbes.com/sites/forbeshuma nresourcescouncil/2019/09/05/minimizing-employee-turnoverin-a-changing-workforce/?sh=18878afe3884. Accessed 11 Jan 2022

24. Ayyala RS, Taylor GA, Callahan MJ (2020) Stresses and anxieties in the time of the COVID-19 pandemic - what we can learn. Pediatr Radiol 50:1052-1054

25. Valtorta NK, Kanaan M, Gilbody S et al (2016) Loneliness and social isolation as risk factors for coronary heart disease and stroke: systematic review and meta-analysis of longitudinal observational studies. Heart 102:1009-1016

26. Seppälä E, King M (2017) Burnout at work isn't just about exhaustion. It's also about loneliness. Harvard Business Review. https:// hbr.org/2017/06/burnout-at-work-isnt-just-about-exhaustion-itsalso-about-loneliness. Accessed 11 Jan 2022

27. Norbash A, Kadom N (2020) The business case for diversity and inclusion. J Am Coll Radiol 17:676-680

28. Hunt V, Layton D, Prince S (2015) Why diversity matters. McKinsey \& Company. https://www.mckinsey.com/business-functions/ people-and-organizational-performance/our-insights/why-diver sity-matters. Accessed 11 Jan 2022

29. Rock D, Grant H (2016) Why diverse teams are smarter. Harvard Business Review. https://hbr.org/2016/11/why-diverse-teams-aresmarter. Accessed 11 Jan 2022

30. Reynolds A, Lewis D (2017) Teams solve problems faster when they're more cognitively diverse. Harvard Business Review. https://hbr.org/2017/03/teams-solve-problems-faster-when-theyremore-cognitively-diverse. Accessed 11 Jan 2022
31. Welp A, Meier LL, Manser T (2014) Emotional exhaustion and workload predict clinician-rated and objective patient safety. Front Psychol 5:1-13

32. Johnson WB, Smith DG (2021) Male allyship is about paying attention. Harvard Business Review. https://hbr.org/2021/02/maleallyship-is-about-paying-attention. Accessed 11 Jan 2022

33. Thoroughgood CN, Sawyer KB, Webster JR (2020) Creating a trans-inclusive workplace. Harvard Business Review. https://hbr. org/2020/03/creating-a-trans-inclusive-workplace. Accessed 11 Jan 2022

34. Slepian M (2020) Are your D\&I efforts helping employees feel like they belong? Harvard Business Review. https://hbr.org/2020/ 08/are-your-di-efforts-helping-employees-feel-like-they-belong. Accessed 11 Jan 2022

35. Weber J (2019) The roles of allies, mentors and sponsors in employee development. Forbes. https://www.forbes.com/sites/ forbeshumanresourcescouncil/2019/09/27/the-roles-of-alliesmentors-and-sponsors-in-employee-development/?sh $=6$ ceff5f038 ee. Accessed 11 Jan 2022

36. Dobbin F, Kalev A (2016) Why diversity programs fail and what works better. Harv Bus Rev 94:52-59

37. Phan JT (2021) What's the right way to find a mentor? Harvard Business Review. https://hbr.org/2021/03/whats-the-right-way-tofind-a-mentor. Accessed 11 Jan 2022

38. Melaku TM, Beeman A, Smith DG, Johnson WB (2020) Be a better ally. Harvard Business Review. https://hbr.org/2020/11/ be-a-better-ally. Accessed 11 Jan 2022

39. Bredella MA, Fessell D, Thrall JH (2019) Mentorship in academic radiology: why it matters. Insights Imaging 10:107

Publisher's note Springer Nature remains neutral with regard to jurisdictional claims in published maps and institutional affiliations. 